\title{
Projective Motion, Projective Curvature Collineation and Infinitesimal Projective Transformation in a Finsler Space Equipped with Semi-Symmetric Connection S.K. Tiwari, Ved Mani
}

Department of Post-Graduate studies and research in Mathematics K.S. Saket (P.G.) College, Ayodhya Uttar Pradesh, India

\begin{abstract}
Article Info

Volume 7, Issue 5

Page Number: 315-325

Publication Issue :

September-October-2020

Article History

Accepted : 15 Oct 2020

Published : 30 Oct 2020

The present communication has been devoted to the study of projective motion, projective curvature collineation and infinitesimal projective transformation in a Finsler space equipped with semi-symmetric connection. In this communication we have derived results in the form of theorems which hold when the Finsler space under consideration admits both projective motion and projective curvature collineation and in this continuation, we have also derived the relationships which hold when the space under consideration admits a non-affine as well as an affine infinitesimal projective transformation. Keywords : Finsler Space, Projective Motion, Projective Transformation.
\end{abstract}

\section{INTRODUCTION}

Yono [7] introduced the concept of semi-symmetric connection in a Rie- mannian manifold. These connections in local co-ordinate system of Rie- mannian space were introduced by Imai [1], such connections in a Finsler space have been introduced by Mehar and Patel [2], while introducing such a connection in a Finsler space they have noticed that the covariant derivatives of the fundamental function $\mathrm{F}(x, \dot{x})$, the unit vector $l^{i}$ and the directional coordinates $\dot{x}^{i}$ vanish with respect to the semi-symmetric connection while the covariant derivative of the fundamental tensors do not vanish with respect to such a connection. As per these provisions, we write:

$$
\begin{aligned}
& \Pi_{\mathrm{jk}}^{\mathrm{i}}=\mathrm{G}_{j k}^{i}+\mu_{j k}^{i} \quad \text { where, } \\
& \mu_{\mathrm{jk}}^{\mathrm{i}}=\delta_{k}^{i} \nu_{j}-g_{j k} \nu^{i}
\end{aligned}
$$

and call $\Pi_{\mathrm{jk}}^{\mathrm{i}}$ as semi-symmetric connection in a Finsler space $\mathrm{F}_{\mathrm{n}}$. The covariant vector field $v_{j}$ and the contravariant vector field $v^{i}$ are connected by $v_{j}=g_{i j} v^{i}$ Here, it should be noted that the covariant vector $v_{j}$ is not in general homogenious in its directional arguments and accordingly the connection parameters $\Pi_{j k}^{i}$ are 
not homogeneous in its directional arguments, it has also been observed that this connection parameter is not symmetric in its covariant indices. With respect to the connection $\Pi_{j k}^{i}$, the covariant derivative of a vector field $\mathrm{X}^{\mathrm{i}}$ with respect to $\dot{x}^{j}$ to be denoted by $\varsigma_{j} X^{i}$ is defined as :

$$
\varsigma_{j} X^{i}=\dot{\partial}_{j} X^{i}-\left(\dot{\partial}_{m} X^{i}\right) \Pi_{j r}^{m} \dot{x}^{r}+X^{m} \Pi_{j m}^{i} .
$$

This expression of covariant differentiation after taking into account the Berwald's process of covariant differentiation can alternatively be written as:

$$
\varsigma_{j} X^{i}=X_{(\mathrm{j})}^{i}+X^{m} \mu_{j m}^{i}-\left(\dot{\partial}_{m} \mathrm{X}^{i}\right) \mu_{j r}^{m} \dot{x}^{r}
$$

The commutation formula involving the operators $\dot{\partial}_{j}$ and $S_{k}$ for the vector field $X^{i}(x, \dot{x})$ is given according to the following rule:

$$
\left(\dot{\partial}_{j} \varsigma_{k}-\varsigma_{k} \dot{\partial}_{j}\right) X^{i}=X^{s} \Pi_{j k s}^{i}-\left(\dot{\partial}_{s} X^{i}\right) \Pi_{j k r}^{s} \dot{x}^{r}
$$

where $\Pi_{j k r}^{i}=\dot{\partial}_{j} \Pi_{k r}^{i}$ and the term $\Pi_{j k r}^{s} \dot{x}^{\mathrm{r}}$ appearing in (1.5) does not vanish because of the fact that $\Pi_{j k}^{s}$ are not homogeneous in its directional arguments. The repeated application of the process of covariant differentiation as has been given in (1.3) and commutation thereafter gives the following commutation formula for the vector field $X^{i}$

$$
2 \varsigma\left[{ }_{j} \varsigma_{k}\right] X^{i}=X^{h} R_{j k h}^{i}-\left(\dot{\partial}_{m} X^{i}\right) R_{j k h}^{m} \dot{x}^{h}-2\left(\partial_{h} X^{i}\right) \Pi_{[j k]}^{h} \cdot
$$

The curvature tensor type entities $\mathrm{R}_{j k h}^{i}$ appearing in (1.6) are defined as :

$$
R_{j k h}^{i}=2\left\{\partial_{[j} \Pi_{k] h}^{i}-\Pi_{m[k<h>}^{i} \Pi_{j] r}^{m} \dot{x}^{r}+\Pi_{[j<h>}^{m} \Pi_{k] m}^{i}+\Pi_{m h}^{i} \Pi_{[k j]}^{m}\right\} .
$$

where, the indices enclosed in the brackets $<>$ are free from symmetric and skew-symmetric parts.

The entities $\mathbf{R}_{i k h}^{i}$ are quite different from the curvature tensors as have been defined by Rund [5] by the same notation. The contracted curvature tensor type entities $\mathrm{R}_{i k h}^{i}$ satisfy the following :

$$
\begin{array}{ll}
\text { (a) } & \mathrm{R}_{i k h}^{i}=R_{k h} \\
\text { (b) } & R_{k h i}^{i}-2 R_{[\mathrm{hk}]}=2 n L_{[h} U_{k]}
\end{array}
$$

where, the symbol $L_{j} T_{k}^{i}$ are defined by the following rule are

$$
\text { (1.9) } L_{j} T_{k}^{i}=T_{k(\mathrm{j})}^{i}-\left(\dot{\partial}_{j} \mathrm{~T}_{k}^{i}\right) v_{r} \dot{x}^{r}+\left(\dot{\partial}_{m} \mathrm{~T}_{k}^{i}\right) \mathrm{g}_{j r} v^{m} \dot{x}^{r}
$$

The contracted curvature tensor type entities $R_{k h i}^{i}$ can also be expressed in the following alternative form under the assumption $L_{[h} v_{k]}=0$ 


$$
R_{k h i}^{i}=2 R_{[\mathrm{hk}]} .
$$

The curvature tensor type entities $R_{h j k}^{i}$ satisfy the following :

$$
R_{j k h}^{i}+R_{k h j}^{i}+R_{h j k}^{i}=2\left(\delta_{k}^{i} \mathrm{~L}_{[\mathrm{j}} v_{\mathrm{h}]}+\delta_{j}^{i} \mathrm{~L}_{[\mathrm{h}} v_{\mathrm{k}]}+\delta_{h}^{i} \mathrm{~L}_{[\mathrm{k}} v_{\mathrm{j}]}\right)
$$

But, if we take into account the assumption that $L_{[h} v_{k]}=0$ then (1.11) can alternatively be written in the following form

$$
R_{j k h}^{i}+R_{k h j}^{i}+R_{h j k}^{i}=0
$$

\section{LIE-DERIVATIVES OF TENSOR FIELDS, CONNECTION PARAMETERS AND COMMUTATION FORMULAE IN A FINSLER SPACE EQUIPPED WITH SEMI SYMMETRIC CONNECTION}

The Lie-derivative of the mixed tensor $T_{j}^{i}(x, \dot{x})$ in a Finsler space equipped with semi-symmetric connection is expressible in the form.

$$
£_{v} T_{j}^{i}=\left(\varsigma_{k} \mathrm{~T}_{j}^{i}\right) v^{k}+\left(\dot{\partial}_{k} \mathrm{~T}_{j}^{i}\right)\left(\varsigma_{h} v^{k}\right) \dot{\mathrm{x}}^{h}-T_{j}^{k}\left(\varsigma_{k} v^{i}\right)+\mathrm{T}_{k}^{i}\left(\varsigma_{j} v^{k}\right)
$$

where $v^{i}=v^{i}(x)$ is a contravariant vector field defined over the region $\mathrm{R}$ of the Finsler space equipped with semi-symmetric connection.

We can write the Lie-derivative of the semi-symmetric connection as under

$$
£_{v} \Pi_{j k}^{i}=\varsigma_{j k} v^{i}+\left(\dot{\partial}_{r} \Pi_{j k}^{i}\right)\left(\varsigma_{h} v^{r}\right) \dot{\mathrm{x}}^{h}+v^{h}\left(\varsigma_{h} \Pi_{j k}^{i}-\varsigma_{k} \Pi_{j h}^{i}\right) .
$$

The commutation formula involving the Lie-derivative and partial derivative with respect to directional argument in a Finsler space equipped with semi-symmetric connection is given by :

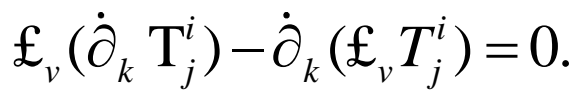

We have the following more related commutation formulae in the Finsler space equipped with semi-symmetric connection.

$$
\begin{aligned}
& £_{v}\left(\varsigma_{k} T_{j}^{i}\right)-\varsigma_{k}\left(£_{v} T_{j}^{i}\right)=T_{j}^{h} £_{v} \Pi_{h k}^{i}-T_{h}^{i} £_{v} \Pi_{j k}^{h}-\left(\dot{\partial}_{h} T_{j}^{i}\right)\left(£_{v} \Pi_{p k}^{h}\right) \dot{x}^{p} \\
& £_{v}\left(\partial_{h} T_{j}^{i}\right)-\partial_{h}\left(£_{v} T_{j}^{i}\right)=0 \\
& \varsigma_{k}\left(£_{v} \Pi_{h j}^{i}\right)-\varsigma_{j}\left(£_{v} \Pi_{h k}^{i}\right)=£_{v} R_{h j k}^{i}+\dot{x}^{l} \Pi_{r h j}^{i} \Pi_{l k}^{r}-\dot{x}^{l} \Pi_{r h k}^{i} £_{v} \Pi_{l j}^{r}
\end{aligned}
$$


where,

$$
\begin{gathered}
\text { (a) } \prod_{j k r}^{i}=\dot{\partial}_{j} \Pi_{k r}^{i} \\
\text { (b) } \mathfrak{f}_{v} R_{h j k}^{i}=\left(\varsigma_{l} R_{h j k}^{i}\right) v^{l}-R_{h j k}^{r}\left(\varsigma_{r} v^{i}\right)+R_{r j k}^{i}\left(\varsigma_{h} v^{r}\right)+\mathrm{R}_{r h k}^{i}\left(\varsigma_{j} v^{r}\right) \\
\quad+R_{h j r}^{i}\left(\varsigma_{k} v^{r}\right)+\left(\dot{\partial}_{r} \mathrm{R}_{h j k}^{i}\right)\left(\varsigma_{p} v^{r}\right) \dot{x}^{p} .
\end{gathered}
$$

We now give the following definitions which will be of use in the later discussions.

\section{DEFINITION (2.1)}

The Finsler space equipped with semi-symmetric connection is said to admit an affine motion provided there exists a vector field $\mathrm{v}(\mathrm{x})$ satisfying

(2.8) $£_{v} \Pi_{j k}^{i}=0$,

where $\Pi_{j k}^{i}(x, \dot{x})$ is the semi-symmetric connection

\section{DEFINITION (2.2)}

The Finsler space equipped with semi-symmetric connection is said to be symmetric provided the curvature tensor type entity appearing in (1.7) satisfies

(2.9) $\varsigma_{m} R_{j k h}^{i}=0$.

\section{DEFINITION (2.3)}

The infinitesimal point transformation $\bar{x}^{i}=x^{i}+v^{i}(x) d t$ defines a projective curvature collineation in a Finsler space equipped with semi-symmetric connection provided that the space under consideration admits a vector field $v^{i}(x)$ satisfying

$$
£_{v} R_{j k h}^{i}=0
$$

\section{DEFINITION (2.4)}

The Finsler space equipped with semi-symmetric connection is said to admit a Ricci type projective curvature collineation provided that there exists a vector field $v^{i}(x)$ satisfying

$$
£_{v} R_{k h}=0 \text {, where } R_{i k h}^{i}=R_{k h}
$$




\section{DEFINITION (2.5)}

The infinitesimal point transformation $\bar{x}^{i}=x^{i}+v^{i}(x) d t$ defines an infinitesimal projective transformation in a Finsler space equipped with semi-symmetric connection provided that the Lie-derivative of the semi-symmetric connection has the form [4]

$$
£_{v} \Pi_{j k}^{i}=\delta_{j}^{i} c_{k}+\delta_{k}^{i} c_{j}-g_{j k} g^{i l} p_{l},
$$

where $c_{j}(x, \dot{x})$ and $p_{l}(x, \dot{x})$ are covariant vector fields depending both upon positional and directional arguments and they satisfy the following :
(a) $\dot{\partial}_{h} c=c_{h}$
(b) $\dot{\partial}_{k} c_{h}=c_{h k}$,
(c) $c_{k h} \dot{x}^{h}=c_{k}$
(d) $c_{h} \dot{x}^{h}=c$,
(e) $\dot{\partial}_{h} p=p_{h}$,
(f) $\dot{\partial}_{k} p_{j}=p_{k j}$,
(g) $\mathrm{p}_{\mathrm{kh}} \dot{x}^{h}=p_{k}$, and
(h) $\mathrm{p}_{\mathrm{h}} \dot{x}^{h}=p$

\section{PROJECTIVE MOTION AND PROJECTIVE CURVATURE COLLINEATION IN A FINSLER SPACE EQUIPPED WITH SEMI-SYMMETRIC CONNECTION}

With the help of the commutation formula (2.6), we can write

$$
£_{v} R_{h j k}^{i}=\varsigma_{k}\left(£_{v} \Pi_{h j}^{i}\right)-\varsigma_{j}\left(£_{v} \Pi_{h k}^{i}\right)-\dot{x}^{l}\left[\Pi_{r h j}^{i}\left(£_{v} \Pi_{l k}^{r}\right)-\Pi_{r h k}^{i}\left(£_{v} \Pi_{l j}^{r}\right)\right]
$$

using (2.12) in (3.1), we get

$$
\begin{aligned}
\mathfrak{f}_{v} R_{h j k}^{i}=\varsigma_{k}\left(\delta_{h}^{i} c_{j}\right. & \left.+\delta_{j}^{i} c_{h}-g_{h j} g^{i l} p_{l}\right)-\varsigma_{j}\left(\delta_{h}^{i} c_{k}+\delta_{k}^{i} c_{h}-g_{h k} g^{i l} p_{l}\right) \\
& -\dot{x}^{l}\left[\left(p_{l}-c_{l}\right)\left(\prod_{j h k}^{i}-\prod_{k h j}^{i}\right)+\prod_{l h j}^{i} c_{k}-\prod_{l h k}^{i} c_{j}\right]
\end{aligned}
$$

We shall now carry out our studies under two presuppositions one that the covariant vectors $c_{j}$ and $p_{l}$ appearing in (2.12) are covariant constants and the other that these two covariant vectors are covariant constants and also that the two fundamental tensors $g_{i j}$ and $g^{i j}$ are metrical and therefore under these two suppositions we respectively get the following from (3.2)

$$
\begin{aligned}
\mathfrak{£}_{v} R_{h j k}^{i} & =p_{m}\left[\left(\varsigma_{k} g_{h j}\right) g^{i m}+g_{h j}\left(\varsigma_{k} g^{i m}\right)-g_{h k}\left(\varsigma_{j} g^{i m}\right)\right. \\
& \left.+g^{i m}\left(\varsigma_{j} g_{h k}\right)\right]-\dot{x}^{l}\left[\left(p_{l}-c_{l}\right)\left(\Pi_{j h k}^{i}-\Pi_{k h j}^{i}\right)+\Pi_{l h j}^{i} c_{k}-\Pi_{l h k}^{i} \mathrm{c}_{\mathrm{j}}\right] .
\end{aligned}
$$

and

$$
\left.\mathfrak{£}_{v} R_{h j k}^{i}=\dot{x}^{l}\left[\left(c_{l}-p_{l}\right) \prod_{j h k}^{i}-\prod_{k h j}^{i}\right)+\prod_{l h k}^{i} c_{j}-\prod_{l h j}^{i} c_{k}\right]
$$

We now introduce (2.9) in (3.2), (3.3) and (3.4) and accordingly we get the following respectively 


$$
\begin{aligned}
& \varsigma_{k}\left(\delta_{h}^{i} c_{j}+\delta_{j}^{i} c_{h}-g_{h j} g^{i l} p_{l}\right)-\varsigma_{j}\left(\delta_{h}^{i} c_{k}+\delta_{k}^{i} c_{h}-g_{h k} g^{i l} p_{l}\right)- \\
& \dot{x}^{l}\left[\left(p_{l}-c_{l}\right)\left(\prod_{j h k}^{i}-\prod_{k h j}^{i}\right)+\prod_{l h j}^{i} c_{k}-\prod_{l h k}^{i} c_{j}\right]=0 .
\end{aligned}
$$

$$
\begin{aligned}
& p_{m}\left[\left(\varsigma_{k} g_{h j}\right) g^{i m}+g_{h k}\left(\dot{\zeta}_{K} g^{i m}\right)-\mathrm{g}_{\mathrm{hk}}\left(\varsigma_{j} g^{i m}\right)+g^{i m}\left(\varsigma_{j} g_{h k}\right)\right] \\
& -\dot{x}^{l}\left[\left(p_{l}-c_{l}\right)\left(\Pi_{j h k}^{i}-\Pi_{k h j}^{i}\right)+\Pi_{l h j}^{i} c_{k}-\Pi_{l h k}^{i} c_{j}\right]=0 .
\end{aligned}
$$

and

$$
\dot{x}^{l}\left[\left(\mathrm{c}_{l}-p_{l}\right)\left(\prod_{j h k}^{i}-\prod_{k h j}^{i}\right)+\prod_{l h k}^{i} c_{j}-\prod_{l h j}^{i} c_{k}\right]=0
$$

We shall now allow a contraction in (3.5), (3.6) and (3.7) with respect to the indices $i$ and $j$ and as a result of contraction, we respectively get the following:

$$
\begin{aligned}
& \varsigma_{k}\left(n c_{h}-p_{h}\right)-\varsigma_{h} c_{k}-\varsigma_{i}\left(g_{h k} g^{i m} p_{m}\right)-\dot{x}^{l}\left[\left(\mathrm{c}_{l}-p_{l}\right)\left(\prod_{k h i}^{i}-\prod_{i h k}^{i}\right)\right. \\
& \left.+\prod_{l h i}^{i} c_{k}-\prod_{l h k}^{i} c_{i}\right]=0 . \\
& p_{m}\left[g^{i m}\left(\varsigma_{k} g_{h i}+\varsigma_{i} g_{h k}\right)+\mathrm{g}_{\mathrm{hi}}\left(\varsigma_{k} g^{i m}\right)-g_{h k}\left(\varsigma_{i} g^{i m}\right)\right] \\
& -\dot{x}^{l}\left[\left(p_{l}-c_{l}\right)\left(\prod_{i h k}^{i}-\prod_{k h i}^{i}\right)+\prod_{l h i}^{i} c_{k}-\prod_{l h k}^{i} c_{i}\right]=0 .
\end{aligned}
$$

and

$$
\dot{x}^{l}\left[\left(p_{l}-c_{l}\right)\left(\prod_{k h i}^{i}-\prod_{i h k}^{i}\right)+\prod_{l h k}^{i} c_{i}-\prod_{l h i}^{i} c_{k}\right]=0 .
$$

With all such findings in hand, we can therefore state the following :

THEOREM (3.1) :

In a Finsler space equipped with semi-symmetric connection admitting both projective motion and projective curvature collineation respectively characterized by (2.12) and (2.9), (3.8) necessarily holds.

\section{THEOREM (3.2) :}

In a Finsler space equipped with semi-symmetric connection admitting both projective motion and projective curvature collineation respectively characterized by (2.12) and (2.9), (3.9) necessarily holds provided the covariant vectors $c_{j}(x, \dot{x})$ and $p_{j}(x, \dot{x})$ be assumed to be covariant constants. 
THEOREM (3.3) :

In a Finsler space equipped with semi-symmetric connection admitting both projective motion and projective curvature collineation respectively characterized by (2.12) and (2.9), (3.10) necessarily holds provided the covariant vectors $c_{j}(x, \dot{x})$ and $p_{j}(x, \dot{x})$ be assumed to be covariant constants and also that the two fundamental tensors $g_{i j}$ and $g^{i j}$ be assumed to be metrical. We now propose to contract (3.2) with respect to the indices $i$ and $h$ and as a result of this contraction, we get the following after making use of (1.8a)

$$
\begin{array}{r}
£_{v} R_{j k}=\varsigma_{k}\left[(n+1) c_{j}-p_{j}\right]-\varsigma_{j}\left[(n+1) c_{k}-p_{k}\right] \\
-\dot{x}^{l}\left[\left(\mathrm{c}_{1}-\mathrm{p}_{1}\right)\left(\prod_{k i j}^{i}-\prod_{j i k}^{i}\right)+\prod_{l i j}^{i} c_{k}-\prod_{l i k}^{i} c_{j}\right] .
\end{array}
$$

Therefore, we can state :

\section{THEOREM (3.4) :}

In a Finsler space equipped with semi-symmetric connection admitting both projective motion and projective Ricci collineation characterized by (2.12) and (2.11) respectively then we shall always have

$$
\begin{gathered}
\varsigma_{k}\left[(n+1) c_{j}-p_{j}\right]-\varsigma_{j}\left[(n+1) c_{k}-p_{k}\right]-\dot{x}^{l}\left[\left(c_{l}-p_{l}\right)\left(\Pi_{k i j}^{i}-\Pi_{j i k}^{i}\right)\right. \\
\left.+\left(\prod_{l i j}^{i} c_{k}-\prod_{l i k}^{i} c_{j}\right)\right]=0 .
\end{gathered}
$$

Let us now suppose that the Finsler space under consideration admits an affine motion and therefore with the help of (2.12) we can state the following :

\section{THEOREM (3.5) :}

If the Finsler space equipped with semi-symmetric connection admits an affine motion then the covariant vectors $b_{j}(x, \dot{x})$ and $p_{l}(x, \dot{x})$ appearing in (2.12) must separately vanish.

\section{INFINITESIMAL PROJECTIVE TRANSFORMATION IN A FINSLER SPACE EQUIPPED WITH SEMI- SYMMETRIC CONNECTION :}

We allow a transvection in (3.2) by $\dot{x}^{\mathrm{j}} \dot{x}^{\mathrm{k}}$ and thereafter use (2.13) and get

$$
\begin{aligned}
\mathscr{£}_{v} R_{h j k}^{i} & \dot{x}^{j} \dot{x}^{k}=\varsigma_{k}\left[c\left(\delta_{h}^{i} \dot{x}^{k}+\delta_{h}^{i} \dot{x}^{k}\right)-g_{h j} g^{i m} p_{m} \dot{x}^{j} \dot{x}^{k}\right] \\
& -\varsigma_{j}\left[\left(c \delta_{h}^{i} \dot{x}^{j}\right)+c_{h} \dot{x}^{i} \dot{x}^{j}-g_{h k} g^{i m} p_{m} \dot{x}^{j} \dot{x}^{k}\right] \\
& -\dot{x}^{l}\left[\left(\mathrm{p}_{1}-\mathrm{c}_{1}\right)\left(\left(\Pi^{i} j h k-\prod_{k h j}^{i}\right)\right] \dot{x}^{j} \dot{x}^{k}+c\left(\prod_{l h j}^{i} \dot{x}^{j}-\Pi_{l h k}^{i} \dot{x}^{k}\right)\right] .
\end{aligned}
$$

We now allow a contraction in (3.2) with respect to the indices $i$ and $h$ and thereafter use (1.8a) and (2.13) and get

$$
£_{v} R_{j k}=\varsigma_{k}\left[(n+1) c_{j}-p_{j}\right]-\varsigma_{j}\left[(n+1) c_{k}-p_{k}\right]
$$




$$
-\dot{x}^{l}\left[\left(p_{l}-c_{l}\right)\left(\Pi_{j i k}^{i}-\Pi_{k i j}^{i}\right)+\left(\Pi_{l i j}^{i} c_{k}-\Pi_{l i k}^{i} c_{j}\right)\right]
$$

We now transvect (4.2) by $\dot{x}^{\mathrm{j}} \dot{x}^{\mathrm{k}}$ and thereafter use (2.13) and get

$$
\begin{aligned}
& £_{v} R_{j k} \dot{x}^{j} \dot{x}^{k}=\varsigma_{k}[(\mathrm{n}+1) \mathrm{c}-\mathrm{p}] \dot{x}^{k}-\varsigma_{j}[(\mathrm{n}+1) \mathrm{c}-\mathrm{p}] \dot{x}^{j} \\
&-\dot{x}^{l}\left[\left(\mathrm{p}_{1}-\mathrm{c}_{1}\right)\left(\Pi_{j i k}^{i}-\Pi_{k i j}^{i}\right) \dot{x}^{j} \dot{x}^{k}+c\left(\prod_{l i j}^{i} \dot{x}^{j}-\prod_{l i k}^{i} \dot{x}^{k}\right)\right] .
\end{aligned}
$$

We now propose to eliminate the term $\left(\varsigma_{k} c\right) \dot{x}^{k}$ using (4.1) and

(4.3) and the result of elimination gives the following:

$$
\begin{aligned}
\mathrm{Q}_{h}^{i}= & \frac{\delta_{h}^{i}}{n+1}\left[\{(\mathrm{n}+1) \mathrm{c}-\mathrm{p}\} \dot{x}^{l}-\dot{x}^{l}\left\{\left(\mathrm{p}_{1}-\mathrm{c}_{1}\right)\left(\Pi_{j i k}^{i}-\Pi_{k i j}^{i}\right) \dot{x}^{j} \dot{x}^{k}\right\}+\right. \\
& \left\{\Pi_{l h j}^{i} \dot{x}^{j}-\Pi_{l h k}^{i} \dot{x}^{k}\right\}-\dot{x}^{l}\left[\left(\mathrm{p}_{1}-\mathrm{c}_{1}\right)\left(\Pi_{j h k}^{i}-\Pi_{k h j}^{i}\right) \dot{x}^{j} \dot{x}^{k}+c\left(\Pi_{l h j}^{i} \dot{x}^{j}-\Pi_{l h k}^{i} \dot{x}^{k}\right)\right] .
\end{aligned}
$$

where,

$$
\begin{aligned}
& \mathrm{Q}_{h}^{i}=£_{v} R_{h j k}^{i} \dot{x}^{j} \dot{x}^{k}-\frac{\delta_{h}^{i}}{n+1}\left[£_{v} R_{j k} \dot{x}^{j} \dot{x}^{k}-\left(\varsigma_{k} p\right) \dot{x}^{p}\right] \\
& \quad+\varsigma_{k}\left(g_{h j} g^{i m} p_{m}\right)+\varsigma_{j}\left(c \delta_{h}^{i}\right)-\varsigma_{j} c_{h}+\varsigma_{j}\left(g_{h k} g^{i m} p_{m}\right)
\end{aligned}
$$

We now take into account the projective derivation tensor $W_{j}^{i}(\mathrm{x}, \dot{x})$ and apply the commutation formula given by (2.4) to this tensor and get

$$
\text { (4.6) } £_{v}\left(\varsigma_{k} W_{j}^{i}\right)-\varsigma_{k}\left(£_{v} W_{j}^{i}\right)=W_{j}^{h} £_{v} \Pi_{h k}^{i}-W_{h}^{i} £_{v} \Pi_{j k}^{h}-\left(\dot{\partial}_{h} W_{j}^{i}\right)\left(£_{v} \Pi_{r k}^{h}\right) \dot{x}^{r} .
$$

We now apply (2.12) and (2.13) in (4.6) and get

$$
\begin{aligned}
£_{v}\left(\varsigma_{k} W_{j}^{i}\right)- & \varsigma_{k}\left(£_{v} W_{j}^{i}\right)=\mathrm{W}_{j}^{h} \delta_{k}^{i} c_{h}-W_{k}^{i} c_{j}-W_{j}^{h} g_{h k} g^{i l} p_{l} \\
& +W_{h}^{i} g_{j k} g^{h l} p_{l}-2 W_{j}^{i} c_{k}-\left(\dot{\partial}_{k} W_{j}^{i}\right)+\left(\dot{\partial}_{h} W_{j}^{i}\right) \mathrm{g}_{\mathrm{rk}} \mathrm{g}^{\mathrm{hl}} \mathrm{p}_{1} \dot{x}^{r} .
\end{aligned}
$$

where, we have taken into account the following facts
(4.8)
(a) $\dot{\partial}_{r} W_{j}^{i} \dot{x}^{r}=2 W_{j}^{i}$
(b) $\dot{\partial}_{i} W_{j}^{i}=0$,
(c) $W_{i}^{i}=0$ and $W_{k}^{i} \dot{x}^{k}=0$ 
We now make the assumption that the infinitesimal projective transformation given by (2.12) leaves invariant the semi-symmetric covariant derivative of by $W_{j}^{i}$ i.e. $\varsigma_{k} W_{j}^{i}=0$ and as a result of this assumption, we shall have

(4.9) $£_{v}\left(\varsigma_{k} W_{j}^{i}\right)=0$

Because of (4.9), (4.7) can be expressed in the following alternative form

$$
\begin{aligned}
\varsigma_{k}\left(£_{v} W_{j}^{i}\right)=\mathrm{W}_{k}^{i} & c_{j}-W_{j}^{h} \delta_{k}^{i} c_{h}+W_{j}^{h} g_{h k} g^{i l} p_{l} \\
& +W_{h}^{i} g_{j k} g^{h l} p_{l}+2 W_{j}^{i} \mathrm{c}_{\mathrm{k}}-\left(\dot{\partial}_{k} W_{j}^{i}\right)+\left(\dot{\partial}_{h} W_{j}^{i}\right) \mathrm{g}_{\mathrm{rk}} g^{h l} p_{l} \dot{x}^{r}
\end{aligned}
$$

We now allow a contraction in (4.10) with respect to the indices $\mathrm{i}$ and $\mathrm{k}$ and as a result or this contraction, we get the following after making use of (4.8)

$$
\varsigma_{i}\left(£_{v} W_{j}^{i}\right)=(2-n) c_{i} W_{j}^{i}+W_{j}^{h} p_{h}+W_{h}^{i} g_{j i} g^{h l} p_{l}+\left(\dot{\partial}_{h} W_{j}^{i}\right) g_{r i} g^{h l} p_{l} \dot{x}^{r}
$$

We now allow a transvection in (4.10) by $\dot{x}^{k}$ and get

$$
\begin{aligned}
\varsigma_{k}\left(£_{v} W_{j}^{i}\right) \dot{x}^{k}= & 2 W_{j}^{i} c-W_{j}^{h} c_{h} \dot{x}^{i}+W_{j}^{h} g_{h k} g^{i l} p_{i} \dot{x}^{r} \dot{x}^{k} \\
& +W_{h}^{i} g_{j k} g^{h l} p_{l} \dot{x}^{k}+\left(\dot{\partial}_{h} W_{j}^{i}\right) g_{r h} g^{h l} p_{l} \dot{x}^{r} \dot{x}^{k}
\end{aligned}
$$

We now eliminate the term $W_{j}^{i}$ with the help of (4.11) and (4.12) and get

(4.13) $2 c \varsigma_{i}\left(£_{v} W_{j}^{i}\right)-(2-\mathrm{n}) \mathrm{c}_{\mathrm{i}}\left(£_{v} W_{j}^{i}\right) \dot{x}^{k}$

$$
=(2-\mathrm{n}) \mathrm{c}_{\mathrm{i}}\left[\mathrm{W}_{j}^{h} \mathrm{c}_{\mathrm{h}} \dot{x}^{i}+\left\{\left(\dot{\partial}_{h} \mathrm{~W}_{j}^{i}\right) \mathrm{g}_{r k} \mathrm{~g}^{\mathrm{hl}} \dot{x}^{r}-\mathrm{W}_{h}^{i} \mathrm{~g}_{\mathrm{jk}} \mathrm{g}^{\mathrm{hl}}-\mathrm{W}_{j}^{h} \mathrm{~g}_{\mathrm{hk}} \mathrm{g}^{\mathrm{il}}\right\} \mathrm{p}_{l} \dot{x}^{k}\right] .
$$

In the light of all these observations, we can therefore state the following :

\section{THEOREM (4.1) :}

If the Finsler space equipped with semi-symmetric connection admits a non-affine infinitesimal projective transformation such that the covariant derivative of the projective deviation tensor $W_{j}^{i}(\mathrm{x}, \dot{x})$ remains an invariant then (4.13) necessarily holds.

\section{THEROREM (4.2) :}

If the Finsler space equipped with semi-symmetric connection admits an affine infinitesimal projective transformation such that the covariant derivative of the projective deviation tensor $W_{j}^{i}(x, \dot{x})$ remains an invariant then $Q_{h}^{i}=0$ where $Q_{h}^{i}$ has been given by (4.5)

As a very special case, if we now suppose that the space under consideration is Lie invariant one

$$
\text { (4.14) } £_{v} W_{j}^{i}=0
$$

Then in such a case, we can state : 


\section{THEOREM (4.3)}

If the Finsler space equipped with semi-symmetric connection admits a non-affine infinitesimal projective transformation such that the projective deviation $W^{v}$ is Lie invariant then following holds :

$$
\text { (4.15) }(2-n) c_{i} W_{j}^{h} c_{h} \dot{x}^{i}=c_{i}(2-n)\left[\left\{\left(W_{h}^{i} g_{j k} g^{h l}-\left(\dot{\partial}_{h} W_{j}^{i}\right) g_{r k} g^{h l} \dot{x}^{r}+W_{j}^{h} g_{h k} g^{i l}\right\} p_{l} \dot{x}^{k}\right]\right. \text {. }
$$

At this state, if we assume that the covariant vectors $c_{j}(x, \dot{x})$ and $p_{j}(x, \dot{x})$ appearing in $(2.12)$ are covariant constants then with the help of (4.4) and (4.5), we shall have :

$$
\begin{aligned}
& £_{v} R_{h j k}^{i} \dot{x}^{j} \dot{x}^{k}-\frac{\delta_{h}^{i}}{n+1} £_{v} R_{j k} \dot{x}^{j} \dot{x}^{k}+\varsigma_{k}\left(\mathrm{~g}_{\mathrm{hj}} \mathrm{g}^{\mathrm{im}}\right) \mathrm{p}_{\mathrm{m}}+\varsigma_{j}\left(\mathrm{~g}_{\mathrm{hk}} \mathrm{g}^{\mathrm{im}}\right) \mathrm{p}_{\mathrm{m}} \\
& =\frac{\delta_{h}^{i}}{n+1}\left[\{(\mathrm{n}+1) \mathrm{c}-\mathrm{p}\} \dot{x}^{l}-\dot{x}^{l}\left(\mathrm{p}_{1}-\mathrm{c}_{1}\right)\left(\prod_{j i k}^{i}-\prod_{k i j}^{i}\right) \dot{x}^{j} \dot{x}^{k}\right. \\
& \left.\quad+\left\{\prod_{l h j}^{i} \dot{\mathrm{x}}^{\mathrm{j}}-\prod_{l h k}^{i} \dot{\mathrm{x}}^{k}\right\}\right]-\dot{\mathrm{x}}^{l}\left[\left(\mathrm{p}_{1}-\mathrm{c}_{1}\right)\left(\prod_{j h k}^{i}-\prod_{k h j}^{i}\right) \dot{\mathrm{x}}^{j} \dot{\mathrm{x}}^{k}\right. \\
& \left.\quad+c\left(\prod_{l h j}^{i} \dot{\mathrm{x}}^{\mathrm{j}}-\prod_{l h k}^{i} \dot{\mathrm{x}}^{k}\right)\right] .
\end{aligned}
$$

If we now assume that the Finsler space under consideration admits both projective curvature collineation and projective Ricci collineation then with the help of (4.15), we have

$$
\begin{aligned}
\varsigma_{k}\left(g_{\mathrm{hj}} \mathrm{g}^{\mathrm{im}}\right) \mathrm{p}_{\mathrm{m}}+\varsigma_{j} & \left(\mathrm{~g}_{\mathrm{hk}} \mathrm{g}^{\mathrm{im}}\right) \mathrm{p}_{m}=\frac{\delta_{h}^{i}}{n+1}\left[\{(\mathrm{n}+1) \mathrm{c}-\mathrm{p}\} \dot{x}^{l}\right. \\
& \left.-\dot{\mathrm{x}}^{l}\left(\mathrm{p}_{1}-\mathrm{c}_{1}\right)\left(\prod_{j i k}^{i}-\prod_{k i j}^{i}\right) \dot{\mathrm{x}}^{\mathrm{j}} \dot{\mathrm{x}}^{k}+\left\{\prod_{l h j}^{i} \dot{\mathrm{x}}^{\mathrm{j}}-\prod_{l h k}^{i} \dot{\mathrm{x}}^{k}\right\}\right] \\
& -\dot{\mathrm{x}}^{l}\left[\left(\mathrm{p}_{1}-\mathrm{c}_{1}\right)\left(\prod_{j h k}^{i}-\prod_{k h j}^{i}\right) \dot{\mathrm{x}}^{\mathrm{j}} \dot{\mathrm{x}}^{k}\right. \\
& \left.+\mathrm{c}\left(\prod_{l h j}^{i} \dot{\mathrm{x}}^{\mathrm{j}}-\prod_{l h k}^{i} \dot{\mathrm{x}}^{k}\right)\right] .
\end{aligned}
$$

With the help of (4.16) and (4.17), we can therefore state the following :

\section{THEOREM (4.4) :}

In a Finsler space equipped with semi-symmetric connection (4.15) is always true provided the covariant vectors $c_{j}(x, \dot{x})$ and $p_{j}(x, \dot{x})$ appearing in $(2.12)$ be assumed to be covariant constants.

\section{THEOREM (4.5) :}

In a Finsler space equipped with semi-symmetric connection (4.16) is always true provided the Finsler space under consideration admits both projective curvature collineatiion and projective Ricci collineation and also that the covariant vectors $c_{j}(x, \dot{x})$ and $p_{j}(x, \dot{x})$ appearing in (2.12) be assumed to be covariant constants. 


\section{REFERENCES}

[1]. Imai, T. , Notes on semi-symmetric connection, Tensor (N.S.), 273-276, (1972).

[2]. Mehar, F.M. , Semi-symmetric connections in a Finsler and space, Rend, Acad. Naz. Dei Lincei XL (4), Patel, L.M. 24-25,281-287 (1473/74)

[3]. Mishra, R.B. , A symmetric Finsler space, Tensor (N.S.) 38, 346-350, (1972).

[4]. Pande, H.D. , Special infinitesimal projective transform and ation in a Finsler space, Acad Naz. dei Kumar, A. Lincei Rend.58(3-4), 190-194 (1974).

[5]. Rund, H. , The differential geometry of Finsler spaces, Springer Verlag, Berlin (1959)

[6]. Takano, K. , On projective motion in spaces with birecurrent curvature, Tensor (N.S.) 12, 28-32, (1962).

[7]. Yono, K. , The theory of Lie-derivatives and its applications, North Holland Publishing Co., Amsterdam (1957).

\section{Cite this article as :}

S. K. Tiwari, Ved Mani, "Projective Motion, Projective Curvature Collineation and Infinitesimal Projective Transformation in a Finsler Space Equipped with Semi-Symmetric Connection", International Journal of Scientific Research in Science and Technology (IJSRST), Online ISSN : 2395-602X, Print ISSN : 2395-6011, Volume 7 Issue 5, pp. 315-325, September-October 2020. Available at doi : https://doi.org/10.32628/IJSRST207568 Journal URL : http://ijsrst.com/IJSRST207568 